\title{
Surviving the Zombie Apocalypse: A Population Dynamics Based Approach
}

\author{
L. H. Miranda Filho ${ }^{*} @$, Bruno V. Ribeiro ${ }^{2}$, Paulo M.M. Rocha ${ }^{3}$, Daniel D.A. Santos ${ }^{4}$, Natália C. \\ de Sena ${ }^{5}$ \\ ${ }^{1}$ Universidade Federal Rural de Pernambuco, Departamento de Física, 52171-900, Recife, PE, Brasil. \\ ${ }^{2}$ Instituto Federal de Brasília, 71826-006, Riacho Fundo I, DF, Brasil. \\ ${ }^{3}$ Universidade de Brasília, Instituto de Física e Centro Internacional de Física da Matéria Condensada, 70919-970, Brasília, \\ Brasil. \\ ${ }^{4}$ Instituto Federal de Brasília, 73380-900, Planaltina, DF, Brasil. \\ ${ }^{5}$ Instituto Federal de Brasília, Setor de Múltiplas Atividades, 72429-005, Gama, DF, Brasil.
}

Received on February 22, 2020. Revised on April 16, 2020. Accepted on May 14, 2020.

\begin{abstract}
Living-dead creatures are recurrent in various folk myths and recently became an icon of popular culture. The "zombie-ism" is usually caused by an infectious-like disease that has no cure and ultimately inflicts most of the human population, leading to a Zombie Apocalypse. In this work, we propose an epidemiological model for a zombie outbreak. By introducing an infection parameter, we show that human survival is possible in certain scenarios. Furthermore, our model allows for three distinct dynamical regimes, only one of which accounts for the full blown Zombie Apocalypse. Our results are obtained both for a fully connected time continuous model and for a stochastic individual based approach.
\end{abstract}

Keywords: Zombie dynamics, Infectious diseases, Numerical modelling, Discrete model.

\section{Introduction}

The fear of living-dead creatures seems to be universal in the human imagination. Many different cultures (e.g. Vikings, Middle-eastern, African, Caribbean...) developed, independently, beliefs in characters that were able to rise from the dead and terrorize living people, either in dreams or in real life [1,2].

Nowadays, the term "zombie" |3], widely propagated in literature [4, 5], games [6,7] and cinematography [8, 10], refers precisely to the idea of creatures without selfcontrol, guided by a rampant hunger for human flesh.

From the etymological viewpoint, this word comes from the Haitian folk myth, where it was believed that powerful sorcerers (called bokors) would be able to reanimate corpses, turning them into slaves devoid of soul [2].

The modern zombie, however, is very different from its original, folkloric counterpart. Current media depicts zombies with a more science-inspired approach, treating the "zombie-ism" as a disease-like condition [1, 6, 9, usually with no cure.

This may take many forms, from a general condition afflicting all people after death, as in [5], to a contactbased infection, where only people killed by zombies become one [4], and even a spreading virus that kills the host and turns it into a zombie, as depicted in 9 .

${ }^{*}$ Correspondence email address: lucmiranda@gmail.com
It is a common theme in fiction to discuss the events of a "Zombie Apocalypse" [1, 9, a situation in which the zombie condition completely overwhelms society's means to contain it, turning most of the human population into zombies.

In recent years, there have been many attempts to describe this scenario with increasing levels of scientific rigor. Based on epidemiological models such as SIR or SIS [11, 12, that have been successfully adapted in the past to describe other problems 13 15], there have been efforts in the direction of formal population interaction models for zombies 16 18]. The model proposed in 16], that pioneered this research topic from this point of view, describes the Living-Zombie conflict via a three agent population model. A system of coupled differential equations describing the evolutions of Susceptible, Zombie and Removed individuals is presented as a basic model for zombie infection, from which equilibrium and stability are determined. The outcome is illustrated by numerical solutions. This model has been further applied in [17,18. Other models, considering different aspects such as emotional responses, have also been put forward in 19,20 .

A recurring phenomenon in these models is the fatalistic view that a Zombie Apocalypse is inevitable. We propose a model in which this is not always the case. By introducing an infection parameter modelling the direct resurrection of Living as Zombies, we allow for the Zom- 
bies killed by conflict to be permanently removed from the dynamics. With this parameter, not present in previously described models, we find cases in which human survival is possible. We present two different approaches for this idea: a differential- equation-based model and a discrete Monte Carlo agent-based model.

In the next section, we present the rules that lead to the proposed set of differential equations for the LivingZombie interaction. From this, we obtain the equilibrium conditions and recover previous results. Following, the different dynamical regimes of the model are described and illustrated by numerical results. The rules for the discrete model are then presented and the outcomes are analyzed analogously. We remark the differences between survivability regions obtained for both models. We present our final remarks in the conclusion.

\section{The Model and Equilibrium Conditions}

The model proposed here describes the dynamics of a two-species population: the Living one $(L)$, which has given birth and death rates, as in usual population dynamics models, and interacts violently with the Zombie one $(Z)$. This interaction may be favorable to the first species, resulting in the death of Zombies, or to the latter, leading to the transformation of individuals from the first species to the second. Besides interaction, the Zombie population may grow through the death of infected Living individuals. Non-infected individuals that perish and Zombies killed by interaction are labeled as Deceased $(D)$ and do not contribute to the dynamics.

Figure 1 illustrates the rules of the dynamics. Birth and natural death rates are given by the parameters $\alpha$ and $\beta$, respectively. Following the same lines discussed in Chapter 3 of [20], we choose linear birth and death rates in our system of differential equations. In a dynamic governed exclusively by these two parameters, we have exponential solutions with effective growth rate being the difference between them. That is, for $\beta>\alpha$, the population goes to zero and becomes extinct. If $\alpha>\beta$, then we have an indefinite expansion of the population. While for $\alpha=\beta$, the population size remains the same, although this equilibrium situation is unstable. Furthermore, in this work we assume that $\alpha \leq 1$, which implies that, in a unit time step, the Living population will not increase

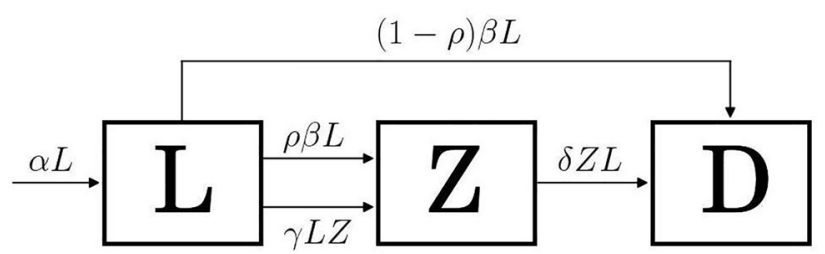

Figure 1: Schematic representation of the model for the system dynamics. more than the existing population. The occurrence of Living-Zombie encounters is assumed to be proportional to the product of the two populations. The rate at which Zombies are generated through interaction is given by the parameter $\gamma$, whilst the rate at which they are killed by interaction is given by $\delta$. The meaning of these last parameters motivate the naming Living strength, for the latter, and Zombie strength, for the former. Finally, we introduce an infection parameter $\rho$, accounting for the fraction of Living that dies due to natural causes and has contracted the "Zombie condition".

The resulting equations for the evolution of the population, including the Deceased, are

$$
\begin{aligned}
\dot{L} & =(\alpha-\beta) L-\gamma L Z \\
\dot{Z} & =\rho \beta L+(\gamma-\delta) L Z \\
\dot{D} & =(1-\rho) \beta L+\delta L Z
\end{aligned}
$$

According to these rules, the equation for the Deceased is decoupled from the $(L, Z)$ evolution.

Given the pair of equations governing the evolution of the Living-Zombie population (first and second equations in (1)), it is possible to analyze the stability of the dynamics. The first step towards this is to obtain the fixed points of the system.

One trivially finds that $L=0$ is a set of fixed points for any value of $Z$ and the parameters. This scenario corresponds to a "Zombie Apocalypse", where the Living species is extinct and the Zombie population ceases to grow, similar to the 'doomsday' equilibrium of 16$]$.

There is a second set of fixed points for any $L \neq 0$, $\gamma \neq 0$ and $\gamma \neq \delta$ at

$$
Z^{*}=\frac{\alpha-\beta}{\gamma}=\frac{\rho \beta}{\delta-\gamma} .
$$

This set is non-hyperbolic. Note that only the cases with $\delta>\gamma$ give non-negative values for $Z^{*}$. The value of $Z^{*}$ defines two regions of distinct behaviour in which the Zombie population remains constant: while in one region the Living population grows, in the other it decreases. The criteria defining these two behaviours are

$$
\begin{aligned}
& Z_{c}=\frac{\rho \beta}{\delta-\gamma} \text { and } \frac{\alpha-\beta}{\gamma}<\frac{\rho \beta}{\delta-\gamma} \rightarrow \dot{L}<0 \\
& Z_{c}=\frac{\rho \beta}{\delta-\gamma} \text { and } \frac{\alpha-\beta}{\gamma}>\frac{\rho \beta}{\delta-\gamma} \rightarrow \dot{L}>0 .
\end{aligned}
$$

From these conditions, we can infer that coexistence of the two species - Living population either constant or growing along with constant positive Zombie population - is only possible if $\rho$ and $\beta$ are non-zero, that is, nonvanishing infection and Living death rates are mandatory. Also, to guarantee the finiteness and positiveness of $Z_{c}$, the Living strength parameter $\delta$ must be strictly greater than the Zombie strength parameter $\gamma$, which means that birth rate alone does not seem to be enough to placate the Zombie apocalypse. 
In Figure 2, we show the parameter sub-space where $\beta=0.2$ and $\gamma=0.6$ for different values of $\rho$. The hyperbolas represent the case of Competition (2), in which both populations stabilize. For each value of $\rho$, the region between the curves represents the case of Apocalypse (3), in which the Living population asymptotically goes to zero, stabilizing the Zombie population. The remaining region corresponds to Supremacy (4), where the Living population grows without bound. Note that, for greater values of $\rho$, the Supremacy region becomes ever narrower, but still existent in the worst-case scenario of generalized infection $(\rho=1.0)$ amongst all Living. As stated before, the region with $\delta<\gamma$, delimited by the vertical line in 2 , yields a negative value for $Z_{c}$, therefore Competition is not possible here.

Note that for regimes in which there is no birth nor natural death $(\alpha, \beta=0)$, this model reduces to the SZR model presented in 17. It is apparent that, with these parameters, the only set of fixed points are those in which either $L=0$ or $Z=0$, therefore there can be no coexistence. Figure 3 illustrates the behaviour of the model in this setting.

\section{Dynamical Regimes of the Model}

In order to further investigate the elements of the model, we must now proceed to the analysis of actual solutions. As stated above, there are three possible outcomes: Competition, in which both populations do not vanish nor present unbounded growth; Apocalypse, in which the Living population goes to zero; Supremacy, in which the Living population grows without bounds. These behaviours are illustrated, respectively, in Figures 4. 5 and 6 .

In each figure, we present two different sets of parameters and initial conditions, leading to the same qualitative

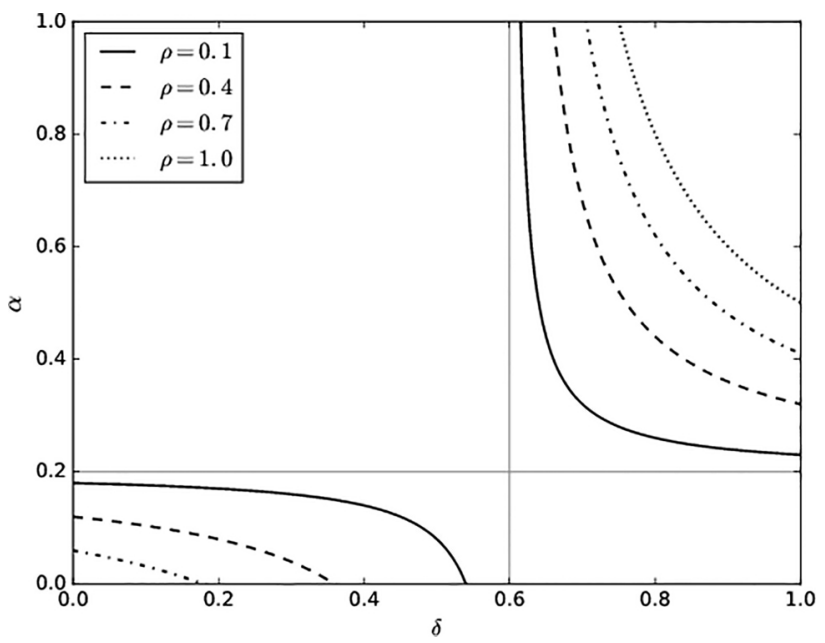

Figure 2: $\alpha=\alpha(\delta)$ as a solution of equation 2 for different values of $\rho$. The horizontal line represents the fixed value of $\beta=0.2$ and the vertical one corresponds to $\gamma=0.6$.

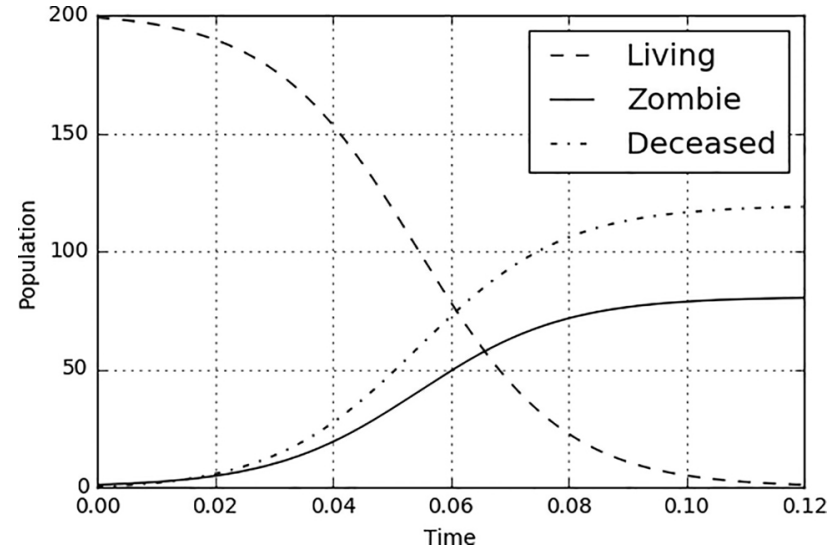

Figure 3: Numerical solution to equations (1) with parameters $\alpha=0, \beta=0, \gamma=1, \delta=0.6$. Initial conditions are $L=199$, $Z=1$.

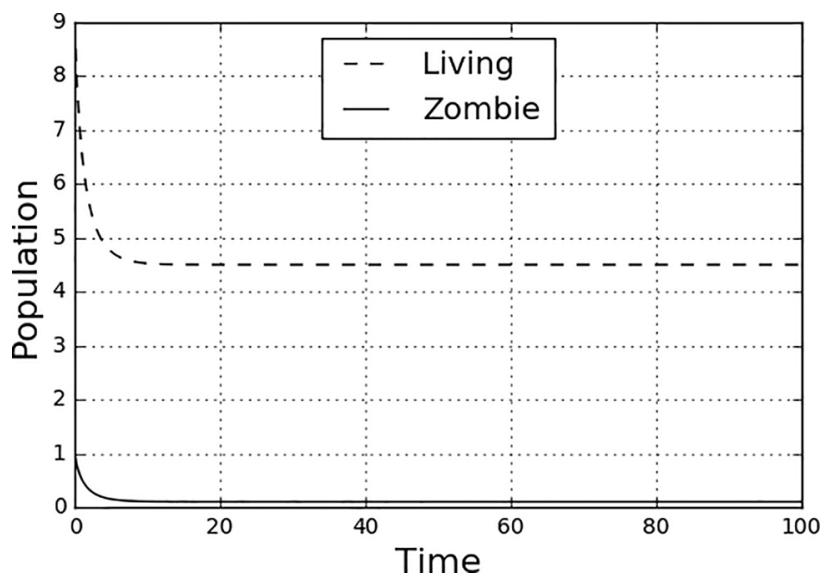

(a)

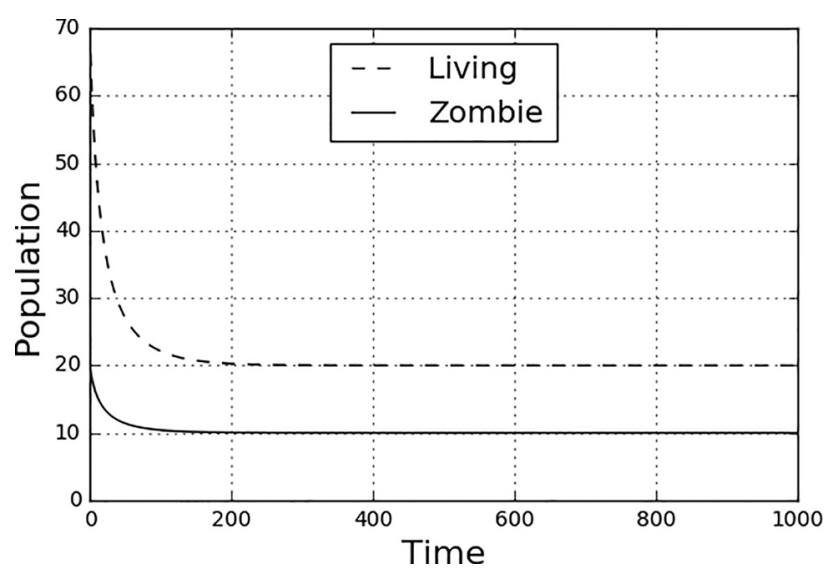

(b)

Figure 4: Numerical solutions to equations (1) with parameters $\alpha=0.15, \beta=0.1$ and $\rho=0.1(\mathrm{a}) \gamma=0.5, \delta=0.6$ and initial conditions $L=9, Z=1$; (b) $\gamma=0.005, \delta=0.006$ and initial conditions $L=70, Z=20$. These cases illustrate Competition. 


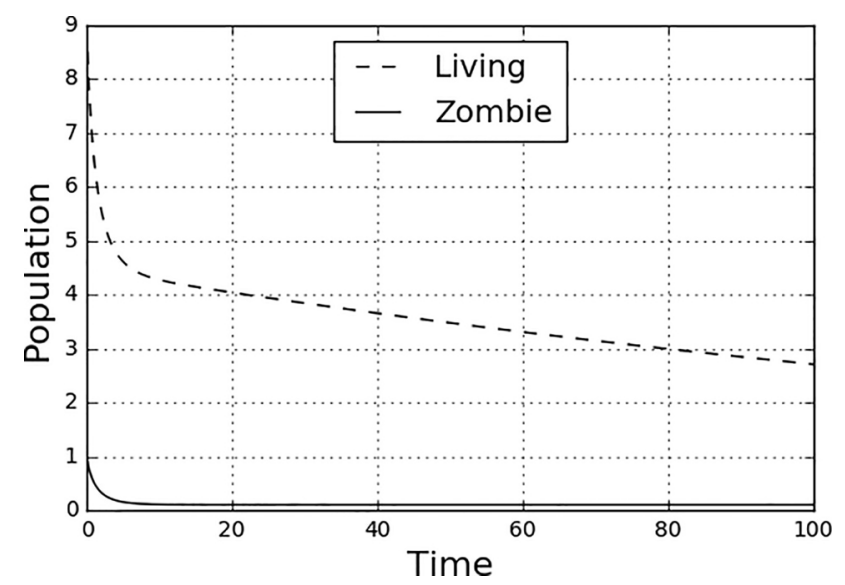

(a)

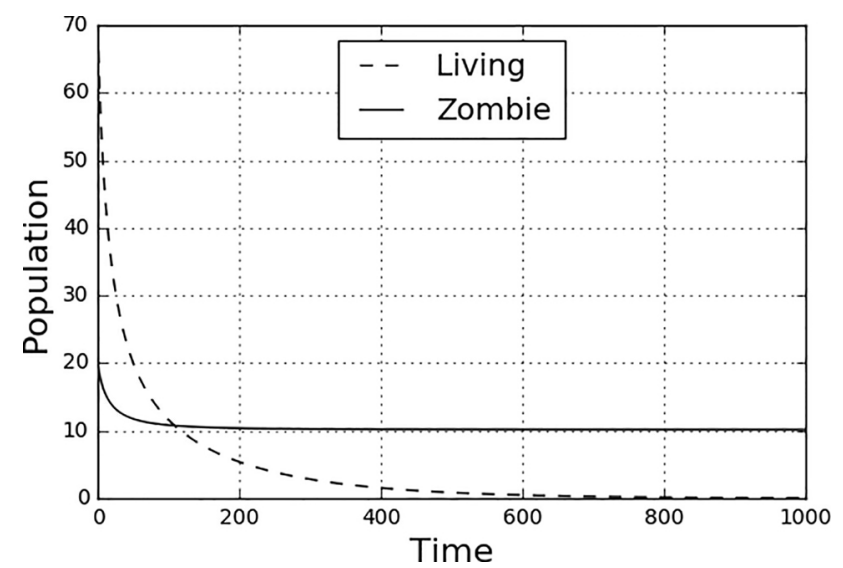

(b)

Figure 5: Numerical solutions to equations (1) with parameters $\alpha=0.145, \beta=0.1$ and $\rho=0.1(\mathrm{a}) \gamma=0.5, \delta=0.6$ and initial conditions $L=9, Z=1$; (b) $\gamma=0.005, \delta=0.006$ and initial conditions $L=70, Z=20$. These cases illustrate Apocalypse.

behaviours. In Figures 4 - $6\left[(\mathrm{a}), Z_{c}=0.2\right.$, therefore initial conditions were chosen in a such a way to guarantee that the Zombie population stabilizes at this value. The same approach was used in Figures 4-6(b) for which $Z_{c}=10$. As stated in 17, we can choose a relevant population size $N$ and reinterpret $L$ and $Z$ as fractions of $N$. By doing this and rescaling the interaction parameters by $\gamma^{\prime}=N \gamma$ and $\delta^{\prime}=N \delta$, the equations remain the same, such that population units are arbitrary.

As observed above, small differences in the parameters may result in different regimes. Living survivability is then a matter of obtaining a "proper" set of parameters. To better represent which sets are favorable, we present Figure 7, in which the color scale represents, for various values of $\alpha$ and $\delta$, the fraction of the populations difference as an indicator of which regime the dynamics follows. As expected from the fixed point analysis, the Apocalypse case given by inequality (3) results in a Living population vanishing asymptotically, thus our indicator assumes negative values at the end of the numerical solution of equations (1). In the same fashion,

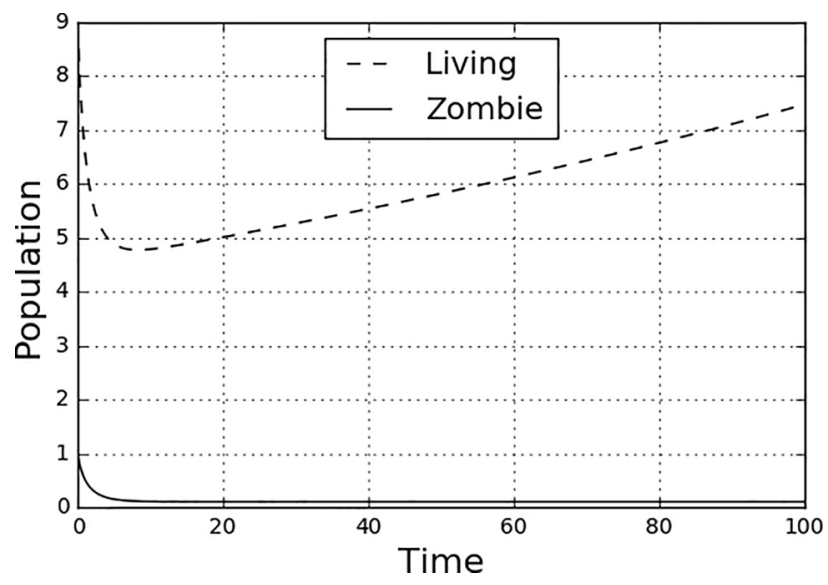

(a)

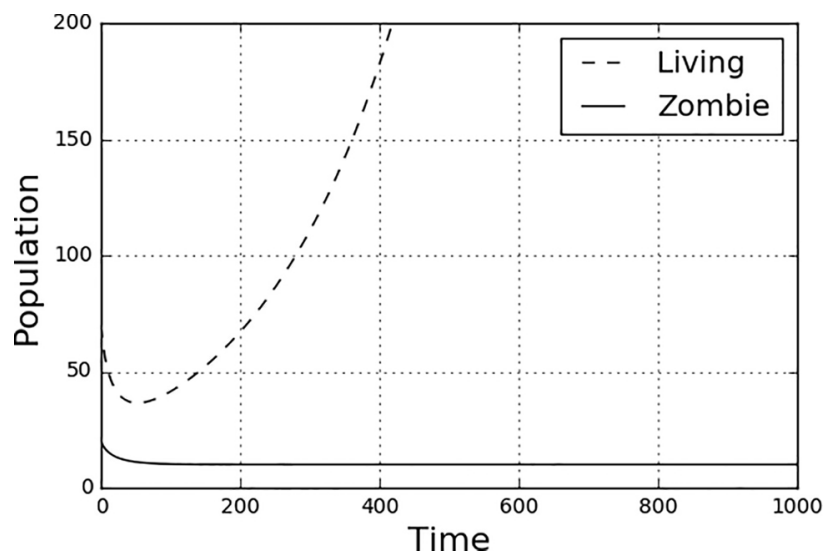

(b)

Figure 6: Numerical solutions to equations (1) with parameters $\alpha=0.155, \beta=0.1$ and $\rho=0.1(\mathrm{a}) \gamma=0.5, \delta=0.6$ and initial conditions $L=9, Z=1$; (b) $\gamma=0.005, \delta=0.006$ and initial conditions $L=70, Z=20$. These cases illustrate Supremacy.

for the Supremacy case given by (4), the Living population will grow exponentially, resulting in a positive value for the indicator. These parameters are chosen for being related to the Living population and not dependant upon external factors, such as infectability or strength of Zombies.

From the figure we observe, for fixed $\beta, \gamma$ and $\rho$, two distinct regimes. These are separated by a narrow region centered in the curve $\alpha=\alpha(\delta)$ obtained from equation (2). One clearly sees, in this figure, that Living survival is less likely event, as a larger area is favorable to Zombie victory. This phenomenon is especially visible when $\delta<\gamma$, case in which Zombie victory is guaranteed, regardless of Living birth rate.

\section{Discrete Model}

The problem can also be described by an alternative approach based on Monte Carlo methods, where we consider interactions in a discrete manner. This is done for various reasons, among which are the need for a more "realistic" 


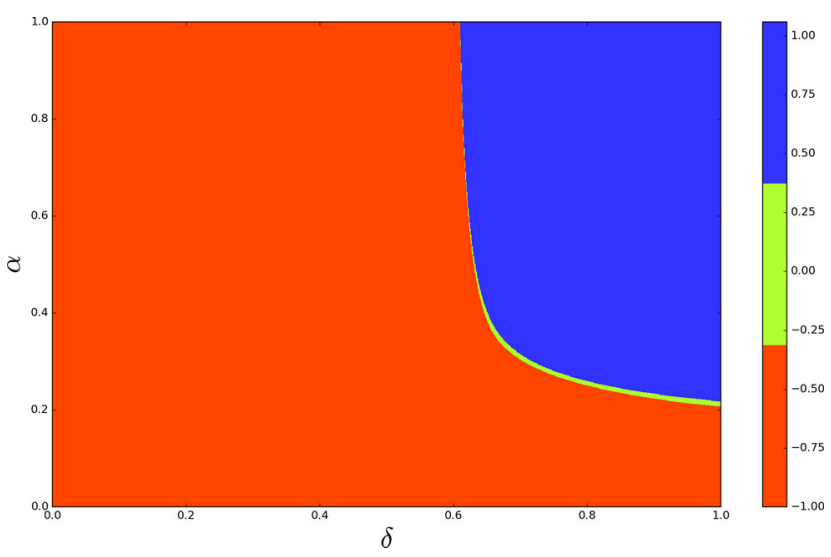

Figure 7: Space of parameters $\alpha$ and $\delta$, with $\beta=0.2, \gamma=0.6$ and $\rho=0.1$. The color scale indicates the value of the fraction of the populations difference $(L-Z) /(L+Z)$ at $t=10^{6}$, with time step $10^{-4}$ and initial conditions $L=0.5, Z=0.25$.

description of the interactions, future implementation of different dynamical ingredients, as well as observation of fluctuation effects due to the finite size of systems. This approach has the added benefit of standardizing the results with the already existing literature [17].

In the analytical model, the existence of sets of critical parameters implies sharp transitions between regimes, which is not expected to be the case in a finite-discrete model. With the express intent of understanding the population dynamics behaviour in a "real" system in the vicinity of these parameters, we propose a simulation in which each individual is labeled as one of two kinds: Living or Zombie. Here, $L$ and $Z$ stand for the sum of all Living and Zombie individuals, respectively. The remaining parameters in the equations (birth and death rates, infection and strengths) are treated as probabilities. From the differential equations, the rate of change of the population depends on the concentrations of Living and Zombies. Computationally, these concentrations are interpreted as the probability of randomly choosing an individual of a given species amongst the total population. Thus, the probability of each event is as given:

$$
\begin{aligned}
& \text { Birth: } P(\text { birth })=P(\text { reproduce } \wedge \text { L pick }) \\
& =\alpha \cdot\left(\frac{L}{N}\right)
\end{aligned}
$$$$
\text { Death: } P(\text { death })=P(\text { die } \wedge \text { L pick })=\beta \cdot\left(\frac{L}{N}\right)
$$$$
\text { Infection: } P(\text { infection })=P(\text { be infected } \wedge \text { death })
$$$$
=\rho \cdot \beta \cdot\left(\frac{L}{N}\right)
$$

$$
\text { Interaction: } P(\mathrm{~L} \text { success) }
$$$$
=P(\mathrm{~L} \text { succes } \wedge(L, Z) \text { pick })=\delta \frac{L \cdot Z}{N^{2}}
$$$$
P(\mathrm{Z} \text { success })=P(\mathrm{Z} \text { succes } \wedge(L, Z) \text { pick })=\gamma \frac{L \cdot Z}{N^{2}}
$$

where $N=L+Z$ is the total population immediately before the event.

Given this set of rules, the simulation consists of a first stage, in which the initial population of Living and Zombie agents is created, followed by a second stage that is divided in three steps: interaction, birth and death/infection. From this point forward, the simulation is played out in turns, repeating the second stage and updating itself until either the Living population is zero or the maximum number of time steps has been reached.

The initial population is set by two parameters: total initial population $\left(N_{0}\right)$ and the initial Living concentration $\left(p_{0}\right)$. Each of the $N_{0}$ individuals are labeled as Living with probability $p_{0}$ randomly. At this stage, the parameters of the dynamics (birth $\alpha$, death $\beta$, infection $\rho$, Living strength $\delta$ and Zombie strength $\gamma$ ) are also set. It is worth noting that, even though the same symbols are used for the parameters in this approach, they have very different meanings. While in the differential equations these parameters are rates, they are probabilities in the Monte Carlo simulation.

The second stage is organized as follows:

- Interaction: Two individuals are randomly chosen from the total population $(N)$. The interaction occurs only in the cases for which a Living-Zombie pair is formed. The success of the individual in the interaction is related to the parameters $\delta$ and $\gamma$. Living success is defined as killing a Zombie and it happens with probability $\delta$. On the other hand, Zombie success happens when it converts a Living into a Zombie, which occurs with probability $\gamma$. Therefore, the possible results of this interaction are: $\{(L$ success, $Z$ success $) ;(L$ success, $Z$ fail $)$; ( $L$ fail, $Z$ succes $) ;(L$ fail, $Z$ fail $)\}$.

- Birth: An individual is randomly chosen from the total population and, in the case of it being a Living agent, another Living individual is generated with probability $\alpha$.

- Death and infection: An individual is randomly chosen from the total population and, in the case of it being a Living agent, death occurs with probability $\beta$. Once death is confirmed, the probability of the individual being infected and converted into a Zombie is given by $\rho$. Otherwise, it is removed from the dynamics.

We show the averaged evolution of both populations in Figures 8,9 and 10 for different sets of parameters. It is straightforward to note, from Figures 9 and 10 . that some cases related to Apocalypse and Supremacy in the continuous model have qualitative analogous within the framework of the discrete, Monte Carlo model. In Figure 10, the simulation was truncated at 500 time steps to avoid numerical overflow problems. Not all of the behaviours observed in the differential equations have analogues in the discrete model, however. With the chosen set of parameters, small variations in $\alpha(\Delta \alpha \sim$ 


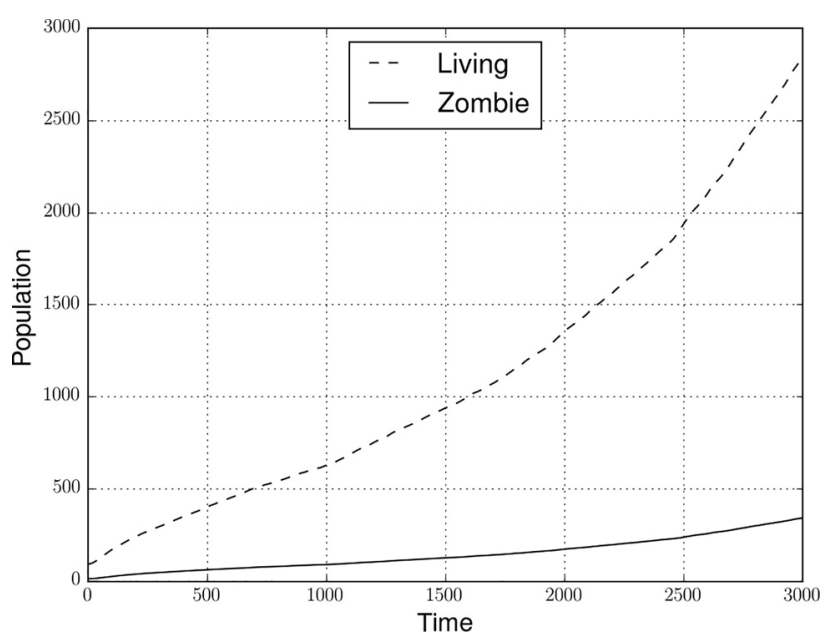

Figure 8: Monte Carlo simulation with parameters $\alpha=0.15$, $\beta=0.1, \gamma=0.5, \delta=0.6, \rho=0.1$. Initial conditions are $N_{0}=100, p_{0}=0.9$ and average over 50 realizations. The simulation was terminated at the maximum number of time steps $t=3000$.

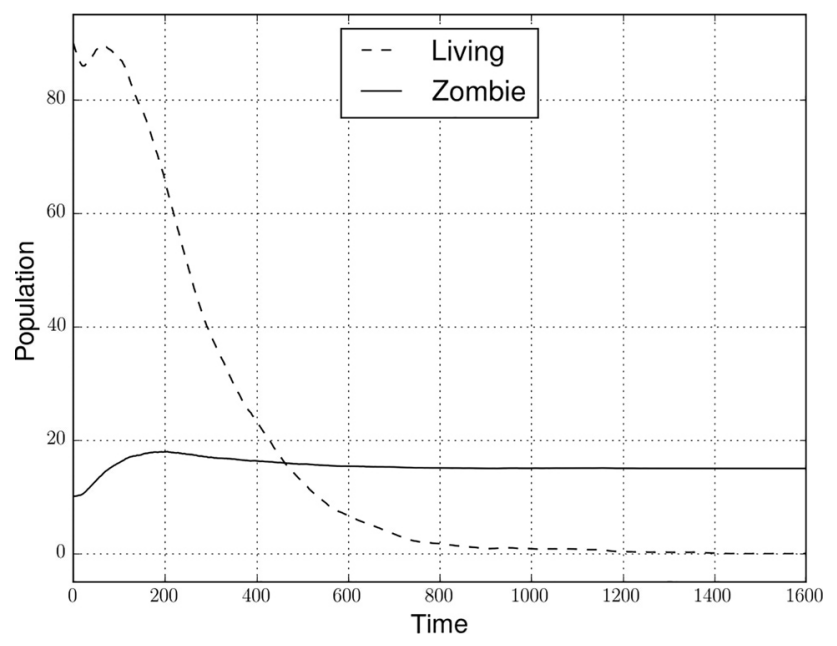

Figure 9: Monte Carlo simulation with parameters $\alpha=0.145$, $\beta=0.1, \gamma=0.5, \delta=0.6, \rho=0.1$. Initial conditions are $N_{0}=100, p_{0}=0.9$ and average over 50 realizations. The simulation was terminated as the Living population reached zero.

$10^{-2}$ ) may cause a substantial change between regimes, due to random fluctuations associated with the events (5). As a consequence, a "coexistence" region, defined as non-vanishing, stabilized populations, is not observable.

To better explore the regimes contained within the discrete model, we build, in Figure 11, a diagram in parameter-space analogous to Figure 7. In this model, we observe a larger Living survival region. The modified discretized dynamics, with a probabilistic interpretation of the parameters, has favored Living survivability. In the large $\alpha$, small $\delta$ region, Living population increases rapidly in the early stages, but decreases drastically as the Zombie population becomes of a comparable size. With the given simulation ruleset, there is no possibility

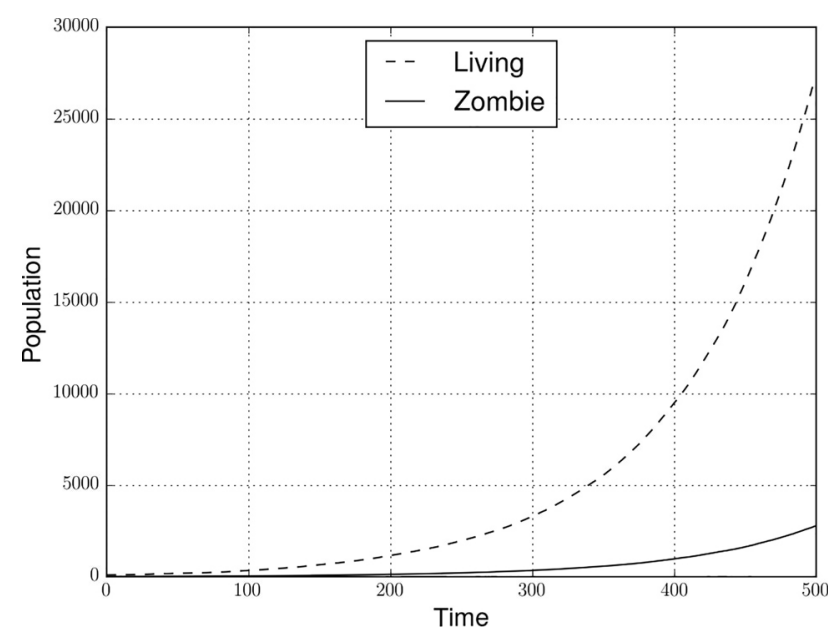

Figure 10: Monte Carlo simulation with parameters $\alpha=0.155$, $\beta=0.1, \gamma=0.5, \delta=0.6, \rho=0.1$. Initial conditions are $N_{0}=100, p_{0}=0.9$ and average over 50 realizations.



Figure 11: Monte Carlo space of parameters $\alpha$ and $\delta$ by setting $\beta=0.2, \gamma=0.6$ and $\rho=0.1$ with 20000 steps. Initial conditions are $N_{0}=150, p_{0}=2 / 3$. The color scale indicates the fraction of the populations difference $(L-Z) /(L+Z)$.

of long term Living survival if $\delta<\gamma$, as in this regime the Living birth rate cannot placate death by confrontation, and subsequent conversion. The apparent "coexistence" in the upper left region is due to finitude of simulation time and is expected to become narrower as simulation times increase.

\section{Conclusion}

In this paper, we presented two different models that describe the dynamics of a Living-Zombie population. On one hand we have a continuous model based on differential equations, in which stability analysis was performed. On the other, a discrete stochastic model was introduced in order to better evaluate to what measure finiteness of the systems affects the outcome. Both models include a parameter related to infection in a non-violent situation, allowing for Living-Zombie conversion without confrontation. The introduction of this parameter, as 
opposed to the model in [16, uncouples the dynamics of the Deceased, reducing the mathematical formulation to two dimensions only.

It is worth noting that system (1) is unbalanced in the sense that the term $\alpha \cdot L$ is not compensated anywhere in the system. This leads to an evolution equation for the total population $\dot{L}+\dot{Z}+\dot{D}=\alpha L(t)$, which is a non-decreasing function of $t$, since $\alpha, L \geq 0$. The focus of the continuous model is on time scales at which the population grows in a Malthusian way. By assuming this, we avoid growth rules which are independent of the instantaneous number of Living, as was proposed by 16 and several models in [20], and thus remove the issue of having their spontaneous emergence.

The linear birth is characteristic of a Holling functional response of Type I and it is used in Lotka-Volterra models. In the seminal work of Holling [21, the linear birth rate saturated at some high value of population density. This constraint has been droped in other works (as in Lotka-Volterra models). We choose to keep the value of $\alpha$ independent of $L$, as we assume that population size is always far from saturation, which makes sense in the context of a Zombie infected world. If there are no zombies and no infection $(Z=0$ and $\rho=0)$, indeed there are three outcomes:

- $\alpha>\beta$, leading to exponentially growing Living population, as it is the case for World Population 22. L only approaches infinity in an infinite time, far beyond the time scale of our model;

- $\beta>\alpha$, resulting in an asymptotically vanishing Living population, which is expected from a population that dies in a higher rate than it reproduces. Again, we remark that $L=0$ is an asymptote. This behaviour is also seen in 16 ;

- $\alpha=\beta$ the system is in an unstable fixed point. The differential equation modelling represents an average behaviour of the system, so it is sensitive to parameter variation. As in the chapter by Munz in [20, in the absence of zombies, the population is modeled by a single linear parameter - in our case, $(\alpha-\beta)$-, representing Malthusian growth.

By analyzing our models, we found three different stationary regimes: Supremacy, Coexistence and Apocalypse. Coexistence is only possible due to the infection parameter, as it was observed not to be possible in [16 17], and only in very specific settings. It is important to note that Supremacy does not imply extinction of the Zombie population, but rather unbounded growth of the Living population with a non-zero, nonetheless controlled, Zombie population. That is to be expected, as in this paper we offer a view of Zombie-ism as an infecto-contagious phenomenon, and as such, can only be eradicated by external agents capable of reducing the infection parameter to zero. As long as this parameter is non-zero, all regimes will always include a non-zero Zombie population.

\section{Acknowledgements}

The authors acknowldege CAPES and CNPq for scholarship during part of this work. Fruitful discussions with T. A. Sobral are gratefully appreciated as well as the design support provided by Daniel Xavier in the production of the posters for XIII Latim American Workshop on Nonlinear Phenomena 2013. The authors, also, thank the participants of the Encontro de Física 2016 for suggestions and the reviewer for comments.

\section{References}

[1] M. Brooks, The Zombie Survival Guide - Complete Protection from the Living Dead (Three Rivers Press, New York, 2003).

[2] E.W. Davis, J. Ethnopharmacol. 9, 85 1983).

[3] Oxford English Dictionary Online, "Zombie", available in https://en.oxforddictionaries.com/definition/ zombie

[4] M. Brooks, World War Z - An Oral History of the Zombie War (Three Rivers Press, New York, 2006).

[5] R. Kirkman, The Walking Dead (Image Comics, Berkeley, 2003-2011).

[6] S. Mikamu, Resident Evil.

[7] K. Inafune, Dead Rising.

[8] G.A. Romero, Night of the Living Dead, available in https://www . youtube. com/watch?v=-_f2Enn8x5s

[9] D. Boyle, 28 Days Later (2002).

[10] S. Pegg and W. Edgar, Shaun of the Dead (2004).

[11] W.O. Kermack and A.G. McKendrick, Proceedings of the Royal Society of London A: Mathematical, Physical and Engineering Sciences 115, 700 (1927).

[12] W.O. Kermack and A.G. McKendrick, Proceedings of the Royal Society of London A: Mathematical, Physical and Engineering Sciences 138, 55 (1932).

[13] H.S. Rodrigues, Int. J. Appl. Math. Comput. Sci. 10, 92 (2016)

[14] S. Rinaldi, F.D. Rossa and P. Landi, Physica A: Statistical Mechanics and its Applications 392, 3231 (2013).

[15] H.S. Rodrigues and M.J. Fonseca, Math. Meth. Appl. Sci. 39, 4780 (2016).

[16] P. Munz, I. Hudea, J. Imad, and R.J. Smith, in Infectious Disease Modelling Research Progress (Nova Science Publishers, Nova York, 2009), p. 133-150.

[17] A.A. Alemi, M. Bierbaum, C.R. Myers and J.P. Sethna, Phys. Rev. E 92, 052801 (2015).

[18] E.T. Lofgren, K.M. Collins, T.C. Smith and R.A. Cartwright, J. Microbiol. Biol. Educ. 17, 137 (2016).

[19] F. Nuñez, C. Ravello, H. Urbina and T. Perez-Acle, arXiv:1210.4469 (2012).

[20] R. Smith, Mathematical Modelling of Zombies (University of Ottawa Press, Ottawa, 2014).

[21] F. Krausmann, S. Gingrich, N. Eisenmenger, K.H. Erb, H. Haberl and M. Fischer-Kowalski, The Canadian Entomologist 91, 293 (1959).

[22] F. Krausmann, S. Gingrich, N. Eisenmenger, K.H. Erb, H. Haberl and M. Fischer-Kowalski, Ecological Economics 68, 2696 (2009). 